\title{
La Web en el móvil: tecnologías y problemática
}

\author{
Por Ángel Hernández-García, Santiago Iglesias-Pradas, Julián Chaparro-Peláez \\ y Félix-José Pascual-Miguel
}

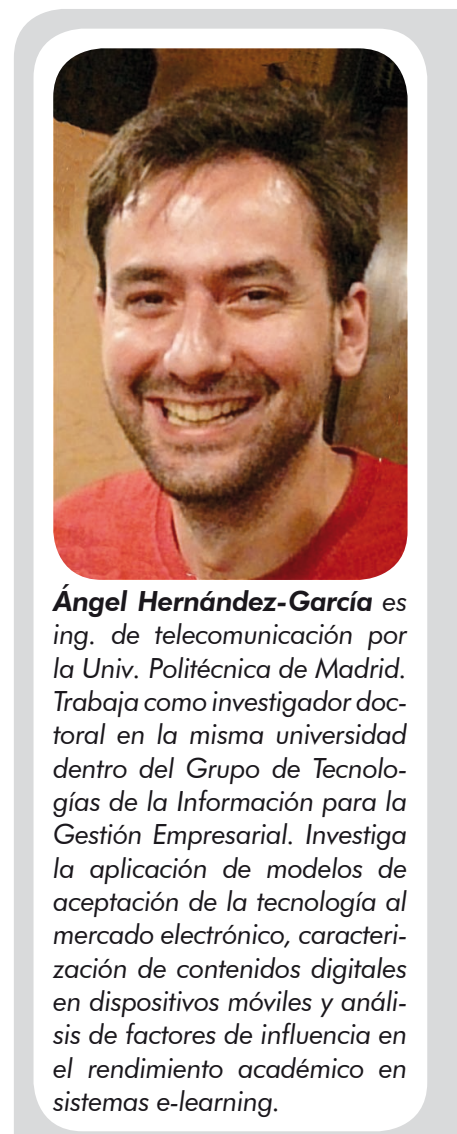

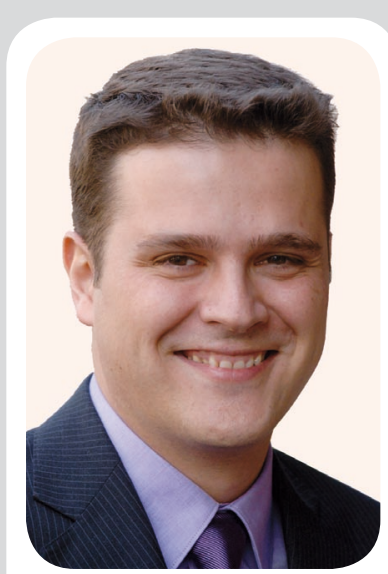

Santiago Iglesias-Pradas es profesor contratado de la ETSI de Telecomunicación de la Univ. Politécnica de Madrid. Doctor ingeniero de telecomunicación y MBA por la UPM. Pertenece al Grupo de Tecnologías de la Información para la Gestión Empresarial y ha participado en distintos proyectos de investigación e innovación con empresas, así como en programas nacionales e internacionales. Es profesor de postgrado en distintas universidades.

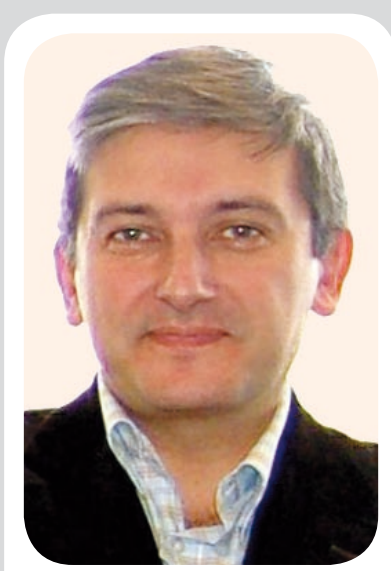

Julián Chaparro-Peláez es profesor titular de la Escuela Técnica Superior de Ingenieros de Telecomunicación (ETSIT) de la Universidad Politécnica de Madrid (UPM). Doctor ingeniero de telecomunicación, ha dirigido diversos proyectos nacionales e internacionales, y participado en numerosos congresos $y$ publicaciones de carácter nacional e internacional.

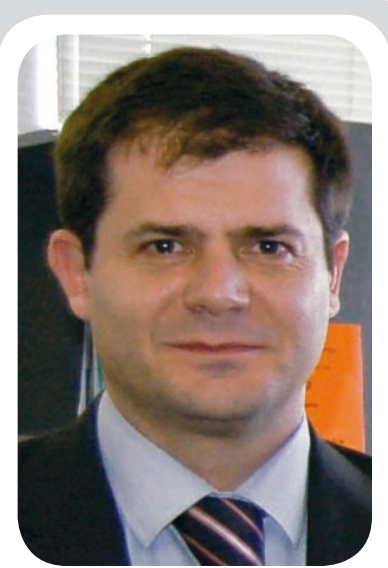

Félix-José Pascual-Migueles profesor de organización de empresas de la Univ. Politécnica de Madrid. Imparte asignaturas de grado y posgrado en las áreas de dirección de empresas y sistemas de información empresariales. Doctor ingeniero de telecomunicación y MBA por la UPM. Ha participado en diferentes proyectos de investigación nacionales e internacionales y es director $y$ secretario académico de tres programas de posgrado de la UPM.

Resumen: En los últimos años se han realizado numerosos intentos de trasladar la experiencia de la navegación de los ordenadores de sobremesa al mundo del móvil, con resultados poco satisfactorios. Sin embargo, las mejoras en las prestaciones de los terminales, así como la aparición de nuevas tecnologías de soporte a la navegación en estos dispositivos están propiciando el surgimiento de una nueva forma de Web móvil (también conocida como Web móvil 2.0). Se estudian las principales características de esta Web móvil 2.0, las barreras para su crecimiento y las posibilidades que ofrecen para su avance tanto las características distintivas de la navegación móvil como las nuevas tecnologías, con especial atención a ajax, los mashups, los widgets y la sindicación de contenidos.

Palabras clave: Teléfonos móviles, Web móvil, Web 2.0, Tecnologías móviles, Web única, Ajax, Mashups, Widgets, Sindicación de contenidos.

\section{Title: Mobile Web: Technologies and issues}

Abstract: In the last years we have seen many attempts to transfer the desktop browser experience to the mobile sphere, with poor results. Nonetheless, both devices' performance improvement and the development of new browsing support technologies are enabling the rise of a new form of mobile Web (also known as Mobile Web 2.0). We detail the characteristics of this mobile Web 2.0, the existing barriers that may impede its growth, as well as the distinctive characteristics of mobile browsing and the various technologies that favour the progress of the mobile Web 2.0, with a special focus in ajax, mashups, widgets and content syndication.

Keywords: Mobile phones, Mobile Web, Web 2.0, Mobile technologies, One web, Ajax, Mashups, Widgets, Content syndication.

Hernández-García, Ángel; Iglesias-Pradas, Santiago; Chaparro-Peláez, Julián; Pascual-Miguel, Félix-José. “La Web en el móvil: tecnologías y problemática". El profesional de la información, 2009, marzo-abril, v. 18, n. 2, pp. 137-144.

DOI: 10.3145/epi.2009.mar.03 


\section{Introducción}

Desde la aparición de la tecnología GSM (Global system for mobile), que impulsó el salto de la telefonía móvil analógica a la digital, el punto de mira de las operadoras se ha centrado en ampliar el volumen del tráfico de datos, más aún teniendo en cuenta el grado de saturación del mercado de tráfico de voz.

Las distintas iniciativas impulsoras del uso de la Web móvil en el pasado, se pueden considerar como interesantes avances técnicos pero auténticos fracasos de mercado debido principalmente a las limitaciones de los terminales y las experiencias negativas de navegación por parte de los usuarios.

Los avances en las capacidades de procesado, almacenamiento y presentación de los contenidos en los terminales móviles, así como el incremento de la velocidad de transmisión propios de la tercera y cuarta generación de telefonía móvil (UMTS o Universal mobile telecommunications systems y HSPA o High-speed packet access) convierten el momento presente en muy propicio para el definitivo despegue de la Web móvil.

http://es.wikipedia.org/wiki/HSPA

\section{Limitaciones y barreras de uso}

Una de las primeras cuestiones que surgen comparando la navegación en el móvil con la navegación desde un ordenador, es la lentitud percibida en el tiempo de carga de los contenidos, que no se debe tanto a la capacidad del enlace como a la latencia, la eventual pérdida de paquetes debida a posibles interferencias en el enlace radio y al tiempo total que tarda un paquete en viajar desde el servidor hasta el cliente más el tiempo que se tarda en mandar una respuesta desde el cliente al servidor, conocido como RTT (round trip tra$v e l$ ), que en redes $3 \mathrm{G}$ es entre 8 y 15 veces más elevado que en la red fija de banda ancha (Rodríguez et al., 2004). Para salvar estos problemas se utilizan soluciones como la descarga en segundo plano de lugares que el usuario posiblemente visite, aunque esta técnica no es muy popular debido a sus inconvenientes: desperdicio de ancho de banda (mayor consumo por tráfico de datos), necesidad de descarga e instalación de aplicaciones [proceso generalmente percibido como molesto por parte de los usuarios (Kleppmann, 2007)] y coste de este tipo de aplicaciones (deben ser específicas para cada plataforma). Otras soluciones pasan por el uso de ajax en versiones móviles de los navegadores más populares (Opera, Internet Explorer, Safari).

Los terminales móviles de mayor potencia, pese a las limitaciones del tamaño de pantalla, duración de la batería y método de entrada de datos, son capaces de procesar tanto el contenido (en lenguaje xhtml) como el estilo (en css) y los complementos (lenguajes inter- pretados como javascript o Python, o plug-ins, como por ejemplo el popular Macromedia Flash) de un sitio web original.

\section{"Pese a los esfuerzos de los fabricantes, los principales problemas de los móviles tienen que ver con la usabilidad y la navegabilidad"}

En muchos casos aún se debe adaptar el contenido al dispositivo móvil. Dentro de las iniciativas de lenguajes de marcado para la creación de webs que puedan ser visualizadas en los dispositivos móviles destaca xhtml MP (Mobile Profile) (OMA, 2008), aprobado por los principales organismos de estandarización (W3C y $O M A$ ) y basado en las mismas etiquetas html usadas en el desarrollo web tradicional. Gracias a esos estándares de lenguajes de marcado los contenidos para internet móvil basados en la Web tradicional, que antes requerían una adaptación, en muchos casos se pueden visualizar perfectamente en terminales móviles. No obstante, debido a las características propias de la interfaz y al comportamiento del usuario, se debe tener en cuenta una serie de factores característicos a la hora de poner en ejecución estos contenidos:

- Interfaz de usuario: el contenido debe adaptarse al tamaño del terminal, que impone un límite en la información que puede presentar al mismo tiempo, mostrando sólo la más relevante. Además, es conveniente reducir al mínimo la entrada de datos mediante el teclado, debido a su incomodidad y lentitud (es aconsejable en este sentido el uso de listas desplegables en el diseño de formularios). La reciente aparición de dispositivos con pantalla táctil palía estas deficiencias.

- Comportamiento del usuario: el uso que se hace del móvil al navegar no tiene por qué ser igual que el de un PC, dado que al llevarse siempre encima se puede acceder a internet en cualquier lugar y tiempo. El tipo de acceso depende del contexto de uso, y se podría diferenciar entre lo que denominaríamos uso "repetitivo" (consulta continua de la misma información, como las cotizaciones bursátiles o previsiones meteorológicas), "de ocio" (consultas que se realizan cuando se dispone de intervalos de tiempo libre, por ejemplo al hacer un viaje en avión o en tiempos de espera -es el perfil de uso más similar al de la navegación web tradicional-) y "urgente" (búsquedas de información concreta y necesaria para llevar a cabo una actividad, por ejemplo, localizar un comercio, adquirir entradas para un espectáculo o buscar rutas hacia un punto de destino). 
Teniendo en cuenta el comportamiento del usuario, los dispositivos móviles ofrecen dos servicios característicos que aportan un valor añadido:

- Personalización del contenido: por lo general, un usuario navegará siempre desde su terminal, considerado como un objeto intransferible, lo que abre la puerta a la personalización de los servicios y contenidos para ese usuario basados en su historial de navegación, horarios, etc.

- Contenidos basados en geolocalización: al poder establecer mediante la Red la ubicación de un usuario cuando navega desde su móvil, el operador puede proporcionar información útil y relevante para él dependiendo de su localización, como por ejemplo información del tráfico, servicios de ayuda en caso de emergencias, ofertas comerciales, predicciones meteorológicas...

\section{"La Web móvil aporta servicios basados en dos valores añadidos: personalización y geolocalización"}

Así pues, aún quedan barreras para el uso generalizado del navegador en el móvil:

- Incluso en los nuevos navegadores móviles de gama alta, pese a los esfuerzos realizados por los fabricantes, los principales problemas siguen teniendo que ver con la usabilidad y la navegabilidad, sobre todo en lo relativo al tamaño de la pantalla y a la aparición del scroll al no caber las páginas enteras.

- Al conectarse desde el móvil a un sitio web normal, no adaptado, el usuario probablemente se encuentre con un buen número de menús y banners superfluos para el contenido al que realmente quiere acceder, ralentizando la navegación, la velocidad de carga y aumentando el coste del tráfico, ya de por sí elevado, en el caso de no utilizar una conexión wifi.

- Como el porcentaje de móviles de gama alta es muy reducido, resulta imprescindible seguir adaptando los contenidos para poder dar servicio a todos. En este sentido, los dominios .mobi representan una iniciativa interesante, puesto que los criterios de diseño y adaptación seguidos garantizan una navegación rápida y sencilla en los dispositivos móviles, proporcionando una buena experiencia de usuario.

\section{La Web móvil 2.0}

Los sitios web están diseñados asumiendo que el usuario tiene una vista general de toda la página y en una pantalla grande los contenidos fundamentales son inmediatamente advertidos. Pero para mostrar webs muy grandes en pantallas pequeñas es necesario adoptar métodos concretos de presentación (Burigat et al., 2008) como el formato estrecho, consistente en la eliminación del molesto scroll horizontal (Nielsen, 2005), o las técnicas de zoom inmersivo. En todo caso, algunos terminales de gama alta, así como los miniordenadores (netbooks) con conexión vía umts, son capaces de mostrar sitios web completos diseñados originalmente para PCs.

\section{http://en.wikipedia.org/wiki/Zooming_user_interface}

Con la llegada del iPhone de Apple se espera una revolución en el campo de internet móvil, y la verdadera eclosión de la Web móvil 2.0. La irrupción de la tecnología de pantalla táctil en el iPhone ha resultado un nuevo paradigma de diseño para los fabricantes (Apple, 2008), y ofrece la posibilidad de visitar las webs en su versión normal y sin cambios de formato. Cuando las empresas comenzaban a llevar a cabo dos versiones de sus sitios web (normal y móvil), el tipo de navegación que propone el iPhone rompe con esa lógica y facilita el acceso a las versiones estándar de los sitios, mejorando notoriamente la usabilidad del sistema y evitando el diseño de una nueva versión móvil (Cobo Romaní; Pardo Kuklinski, 2007). El navegador Safari del iPhone presenta capacidades similares a las del navegador de escritorio, aunque aún se mantienen ciertas limitaciones como el tamaño de pantalla, la instalación de algunos complementos [Flash, por ejemplo, aunque Adobe ha anunciado una nueva versión para iPhone (Perry, 2008)] o tamaño máximo de archivos.

Con esta corriente impulsada por el iPhone se aspira a alcanzar el concepto de one web o web única, cuyos principios fundamentales son los siguientes:

- Una misma dirección web debe ser válida tanto para un ordenador como para un teléfono móvil o una pda.

- La experiencia de un usuario puede variar en diferentes tipos de dispositivo, pero el contenido debe ser homogéneo con independencia del dispositivo.

- La web única no significa la misma web con la misma experiencia de usuario, los mismos elementos de navegación o las mismas funciones en todos los dispositivos. Significa adaptar los contenidos al contexto y a las distintas interfaces.

Para diseñar bajo el marco de la web única es recomendable: a) usar xhtml y css; b) utilizar la información del dispositivo para adaptar el contenido a sus capacidades; c) realizar las pruebas en dispositivos reales.

Por otro lado, el término Web 2.0 hace referencia a un grupo de conceptos, ideas y actitudes que caracterizan una nueva generación de servicios y aplicacio- 
nes disponibles en internet, y que representan una clara evolución desde la Web estática tradicional. Originalmente propuesto por $\mathbf{O}$ 'Reilly en colaboración con $\mathrm{Me}$ diaLive International, el término Web 2.0 se comenzó a utilizar para designar una forma de utilizar y concebir la Web (O'Reilly, 2005), caracterizada por:

- Inteligencia colectiva: la Web se convierte en una red social que se nutre de contenidos mediante una cultura de participación.

- La Web como plataforma: las aplicaciones web tradicionales se convierten en aplicaciones RIA (rich internet applications, o aplicaciones de internet enriquecidas) ejecutadas directamente en el navegador.

\section{"Los operadores móviles no están acostumbrados a colaboraciones en las que el control de la aplicación no esté acotado"}

Hoegg añade al carácter colectivo de la Web la necesidad de servicios para asegurar o valorar la calidad como calificaciones, votaciones, etc., y define la Web 2.0 como "la filosofía de maximizar mutuamente el conocimiento colectivo y valor añadido para cada participante mediante la compartición y creación formal de contenidos generados por el usuario" (Hoegg et al., 2006; De la Torre, 2006).

Es fundamental plantearse qué puede aportar la movilidad a este entorno de colaboración: la ubicuidad del acceso a los servicios. El usuario tiene más flexibilidad para colaborar en esta inteligencia colectiva, puede participar en cualquier momento y desde cualquier sitio, sin la necesidad de un punto fijo de acceso.

En el plano tecnológico es necesario proporcionar internet móvil con atractivos, que ofrezca una experiencia enriquecedora. Por lo tanto, se debe abandonar WAP (wireless application protocol) y la creación de sitios WML (wireless markup language), ya que son un límite para poner en práctica aplicaciones interactivas, y promover el uso de navegadores con capacidades similares o idénticas a los de los ordenadores de sobremesa o portátiles con un doble propósito: permitir la creación de aplicaciones RIA y evitar la creación de réplicas móviles de los sitios web.

Ya se ha empezado a realizar un acercamiento a la Web 2.0 en el entorno móvil. Un ejemplo práctico es la posibilidad que ofrece Google de acceder a sus servicios Google Maps o Gmail desde el móvil. Pero precisamente este ejemplo muestra los impedimentos que plantean las barreras tecnológicas del móvil: el servicio Google Maps para el móvil no se utiliza desde un navegador como en un ordenador de sobremesa. El usuario no ejecuta una aplicación directamente en internet, necesita el paso previo de descarga e instalación de un pequeño cliente específico y compatible para su terminal. Esto presenta una incongruencia significativa. Se tienen terminales con la tecnología necesaria para mostrar los mapas de Google con todo detalle, pero no son capaces de mostrarlos en el navegador.

\section{Tecnologías de soporte a la Web móvil 2.0}

La aparición de la Web 2.0 implica el surgimiento de una serie de nuevas tecnologías y un cambio de modelo, centrado en la mejora de la interactividad de las interfaces de usuario basadas en web (impulsadas por el uso de ajax), la utilización de sistemas de anotación colaborativa o de etiquetado, la sindicación y agregación de contenidos (rss), la combinación de datos y servicios de distintos proveedores (mashups), el enriquecimiento semántico de sitios web mediante la mejora del marcado (microformatos) y el uso de lenguajes de marcado que facilite la independencia de las páginas web con respecto a los terminales móviles que acceden a ellas (W3C, 2008a), como la iniciativa DIAL del $W 3 C$ (W3C, 2007).

\section{"La web única no significa la misma experiencia de usuario y los mismos elementos de navegación"}

\subsection{Ajax}

Ajax (asynchronous javascript and $\mathrm{xml}$ ) es una técnica de desarrollo web que hace posible la ejecución en el navegador manteniendo comunicación asíncrona con el servidor en segundo plano. De esta forma es posible realizar cambios sobre la misma página sin necesidad de recargarla, aumentando la interactividad, velocidad y usabilidad. Además, facilita a los navegadores el acceso directo a aplicaciones web con interfaces similares a las de un ordenador de escritorio. Entre las ventajas de ajax en dispositivos móviles están las siguientes:

1) compatibilidad con los navegadores que soporten los mismos estándares que los de la internet fija;

2) no necesidad de instalación de plug-ins (Mahemoff, 2006), al cargarse y ejecutarse en el navegador (Mesbah; van Deursen, 2008); 
3) economía en la transmisión de datos desde la Red, aspecto de gran importancia en un entorno de ancho de banda limitado.

Entre los inconvenientes se puede destacar el mayor consumo de recursos por parte del cliente, así como la escasez de implantación en los navegadores de terminales de gama media o baja.

Las aplicaciones ajax para un entorno móvil también deberían tener en cuenta que las pérdidas de conexión son más frecuentes, y quizá sería necesario hacer que las aplicaciones fueran más robustas y estuvieran preparadas para estas pérdidas de conexión.

\section{¿Qué aporta ajax a la Web móvil?}

- La comunicación síncrona entre las aplicaciones y el servidor, bajo la forma de petición-respuesta, va en contra de la interactividad, uno de los factores que se pretende potenciar con el nuevo concepto de Web 2.0. La principal aportación de ajax es la posibilidad de reducir el tiempo de respuesta entre las aplicaciones y el servidor gracias a su naturaleza asíncrona, lo que hace posible la solución, en parte, de algunos de los problemas inherentes a las redes móviles como la capacidad y la latencia, mejorando ostensiblemente la interactividad de las aplicaciones.

\section{"Web única significa adaptar los contenidos al contexto y a las interfaces de acceso"}

- Mejora de la usabilidad de los dispositivos móviles en tareas como la introducción de texto (por ejemplo, mediante el uso del texto predictivo, el autocompletado o la validación dinámica de formularios, que favorece una mayor velocidad de entrada de datos reduciendo el número de acciones a realizar por el usuario (Chan; Flaherty, 2005)).

No obstante, la adopción de ajax podría resultar más lenta de lo esperado si no se solucionan los problemas que tienen lugar con las baterías actualmente, ya que la utilización del lenguaje javascript conlleva un consumo de batería muy grande.

En general, dentro de estas aplicaciones sujetas a conexión, las más extendidas son las que sacan partido de la geolocalización. El gps incorporado se va popularizando, aunque todavía está restringido a los equipos de gama alta. El acceso a servicios como Google Maps está permitiendo poner en práctica servicios muy similares a los gps en los que los sistemas de geolocalización se combinan con la capacidad de ubicar al usuario mediante la antena más próxima por la que recibe la señal.
Coincidiendo con el auge de las redes sociales, también se están popularizando los servicios de geotagging, que tienen la capacidad de marcar las coordenadas de una foto para situarla sobre un mapa (Toyama et al., 2003; Torniai et al., 2007).

\subsection{Mashups}

Un mashup (Trevor, 2008), es una aplicación web híbrida de fácil y rápida implementación que usa contenidos de más de una fuente para crear un nuevo servicio completo (Maximilien, 2008). Hace uso de datos y otros servicios de internet en tiempo real, independientemente del mecanismo de comunicación. Los mashups se pueden originar cuando algunos sitios publican APIs (application programming interfaces) que ofrecen a otros sitios la posibilidad de acceder a los datos o servicios subyacentes e integrar ese contenido en sus propios servicios.

Las mashups representan un concepto atractivo e innovador, dado que las empresas pueden utilizarlos para unir las capacidades de aplicaciones complementarias entre sí, superando así la rigidez y las limitaciones de cada aplicación en particular. Si los blogs revolucionaron la publicación en línea, los mashups han revolucionado el desarrollo web, permitiendo que sea posible combinar de forma innovadora, datos procedentes de, por ejemplo, eBay, Amazon, Google, Windows Live o Yahoo.

Los operadores móviles no están acostumbrados a este tipo de colaboraciones en las que el ámbito de control de la aplicación no se encuentra acotado (cualquier aplicación puede hacer uso de otra mediante su API), y en este sentido las iniciativas surgidas son escasas (Open Movilforum, Betavine) por el momento. Las principales dificultades, de naturaleza más cultural que tecnológica, estriban en el cambio hacia el paradigma abierto que requiere la implementación de mashups frente a la tradicional cultura de walled garden (jardín vallado) de los operadores móviles (Chakraborty et al., 2007; Mäkeläinen; Alakoski, 2008). Para explotar el mundo de los mashups deben mostrarse más abiertos a la comunidad de desarrollo y a sus clientes, así como cooperar con compañías que comprendan los ciclos de ejecución, el potencial del marketing viral y los tipos de negocio de la Web 2.0 (Candelon; Arie, 2007)

\section{http://www.open.movilforum.com http://www.betavine.net}

En los terminales móviles son usuales los mashups geográficos. Requieren una fuente cartográfica que pueda proporcionar una presentación visual de la zona involucrada en el proceso; también se necesita una fuente para el tipo de información o datos que contenga el mashup. Los datos se representarán en el mapa de forma gráfica gracias a la propia aplicación. Un ejem- 
plo es el que resulta de la unión de Craigslist con Google Maps; Craigslist es un sitio de clasificados online gratuitos en EUA que ha unido sus fuerzas con Google Maps para crear una nueva forma de elegir un lugar para vivir. El servicio cuenta con un mapa en donde se indican las casas con sus fotos, además de tener publicadas todas las propiedades en un mismo directorio.

\section{http://www.craigslist.com}

Aparte de los geográficos, los tipos más comunes de mashups en los terminales móviles son:

- Noticias. Se agrupan y presentan según las preferencias del usuario: por temas, situadas en un calendario, las que figuran en las primeras páginas de varios medios, etc.

- Vídeos y fotografías. Según los que aportan los proveedores de contenidos o los propios usuarios junto con cualquier otra fuente que pueda proporcionar información ya sea acerca del lugar donde las fotografías o vídeos fueron realizados (geotagging), sobre el autor de los mismos, etc.

- Búsquedas y compras. Se recopilan precios y especificaciones de un mismo producto en diferentes ubicaciones, y los resultados de la búsqueda se comparan y se presentan ordenados para poder elegir el más conveniente.

La adaptación de mashups que funcionan en webs normales a terminales móviles resulta generalmente bastante factible. Basta con un servicio que transforme los datos a un formato que pueda utilizarse en el dispositivo móvil. Un ejemplo de este tipo de servicios es Phonifier Se trata de una herramienta que se instala en el servidor y elimina todo el código innecesario para que pueda ser visualizado fácilmente en un dispositivo móvil. Otros ejemplos de este tipo de servicios son Madgat y el conversor de Google.

\section{http://phonifier.com}

\section{http://www.madgat.com}

http://www.google.com/gwt/n

Aunque el navegador es la plataforma más accesible para elaborar y distribuir un mashup, esto no quiere decir que sea la única y la más apropiada en todos los casos desde la perspectiva del usuario. La presentación de la información en el terminal no se limita por lo tanto a la navegación WAP o web. Pueden utilizarse lectores rss o incluso mensajes sms. Hay servicios basados en sms como Clickatell que ofrecen la posibilidad de enviar mensajes vía http o invocar una petición http mediante el envío de un sms. Por lo tanto, es posible pedir o recibir datos de mashups vía mensajes sms, incluso enviando información al teléfono de otra persona.

http://www.clickatell.com
Otras iniciativas, como $m$ Works, proporcionan una plataforma para crear mashups que se ejecuten como una aplicación en el teléfono móvil, mientras que Yahoo Konfabulator permite crear mashups que se ejecuten como widgets.

http://www.mfoundry.com/platform.html http://widgets.yahoo.com/tools

\subsection{Widgets}

Los widgets son aplicaciones sencillas que ofrecen servicios sobre un sitio web o sobre el escritorio, como calculadoras, diccionarios o titulares de noticias, pudiendo ser personalizados e interactivos. Nokia anunció en abril de 2007 soporte completo a estos widgets (WidSets) en la serie 560 , obteniendo en un mes más de un millón de usuarios registrados (Nokia, 2007a; Nokia, 2007b). Desde un sitio web los usuarios pueden elegir los widgets que descargados aparecerán en sus teléfonos. Una vez situados en el escritorio, reciben información de distintas formas, como vía rss. Por ejemplo, un widget de Google News se ve en forma de un pequeño icono, y al pulsar sobre él, se pueden ver los titulares del sitio web Google News. También hay aplicaciones de este tipo basadas en J2ME (Java 2 micro edition).

Los widgets realizan tareas sencillas de forma muy eficiente. A diferencia de los que están creados para el ordenador personal, los de móvil tratan ahora de popularizar servicios que no habían triunfado por las deficiencias de la Web móvil de los años pasados (Crespo, 2007).

Hasta el momento las compañías elaboran sus propios widgets, por lo que sólo funcionan en determinados terminales o con determinados servicios. Opera dio el primer paso hacia una posible estandarización enviando las especificaciones de uno de ellos al W3C para asegurarse de que funcionan en cualquier plataforma, lo que ha culminado con el desarrollo de un estándar del W3C, aún en estado de borrador (Bersvendsen, 2006; W3C, 2008b). Este paso abre la posibilidad de que las diferentes compañías cooperen para establecer un estándar que todas implementen. De esta manera los desarrolladores podrían dedicarse a crear widgets, dejando al usuario la decisión acerca de en qué plataforma utilizarlos (Munslow Vilar, 2007).

\section{¿Qué aportan los widgets a la Web móvil?}

- Un acceso rápido y continuado a la información. Constituyen una forma sencilla de recibir información útil sin necesidad de navegar (apariencia offline). Teniendo en cuenta que el usuario en movilidad suele caracterizarse por buscar información puntual y muy concreta, y pretende acceder a ella en el menor tiempo posible, esta característica es una gran ventaja. 
- Manejan un volumen menor de datos que el navegador. Sólo necesitan refrescar un poco de información, en lugar de una web completa.

- Su interfaz de usuario está optimizada para tareas generalmente muy específicas. No hay que arrancar el navegador cada vez que se necesite información. Al ser más ligero, el widget se carga mucho más rápido e incluso puede estar integrado en la pantalla principal del teléfono.

- Ofrecen al usuario la posibilidad de añadir funciones al aparato y personalizar el contenido de los propios widgets.

- Están basados en tecnologías familiares para los desarrolladores web como ajax, javascript y css.

\subsection{Sindicación}

Es el proceso mediante el cual, un suscriptor o una red de suscriptores recibe información automatizada sobre la actualización de fuentes de información a las que previamente se ha suscrito (Werbach, 2000).

Hay diferentes formas de acceder o subscribirse a los canales rss: aplicaciones de lectura, agregadores vía web (como Bloglines o Google Reader), plug-ins para navegadores o clientes de correo electrónico, integración en el navegador, servicios web y sistemas de gestión de contenidos con módulos lectores de rss incorporados (como PHP-Nuke, Mambo, o gestores de aprendizaje como Moodle).

\section{¿Qué aporta la sindicación de contenidos a la mo- vilidad?}

Ante todo, reducción de coste por tráfico de datos para el usuario: la sindicación permite acceder a los sitios de interés de una forma más rápida, sabiendo con antelación si los contenidos han sido actualizados; también se pueden descargar los contenidos desde un PC y almacenarlos en el teléfono para leerlos posteriormente sin necesidad de estar conectado.

\section{Conclusiones}

Internet es uno de los mayores activos por explotar en el sector móvil. El impulso para que la Web móvil tenga un éxito mayor depende de la adopción de medidas económico-comerciales y del planteamiento de negocios que posibiliten su expansión, de la superación de las limitaciones aún existentes, y de la potenciación de las capacidades específicas que propicia la movilidad (principalmente la personalización y la geolocalización).

Las principales limitaciones tienen que ver con aspectos como el tamaño de la pantalla, la usabilidad de los dispositivos, el consumo de batería y la necesidad de adecuación del contenido a la Web móvil. Sin embargo, la aparición de nuevos terminales y la madu- ración de nuevas tecnologías como ajax, los mashups, los widgets o la sindicación de contenidos, unido a un cambio de mentalidad orientada a potenciar la creación de una Web única independiente del dispositivo, pueden ayudar a paliar estas deficiencias posibilitando un definitivo despegue de la navegación desde terminales móviles.

Finalmente, todo parece indicar que la próxima generación de la Web móvil girará en torno a las redes sociales y su capacidad de generar contenido para la Red. En esta Web móvil 2.0 los usuarios pasan a tener un papel semejante al de proveedores de contenidos, generando y consumiendo datos en cualquier lugar y momento, por lo que parece necesario un aumento de la velocidad y capacidad de los canales radio (por ejemplo, mediante el uso de tecnologías HSPA) que permita aprovechar todo su potencial.

\section{Referencias}

Apple. iPhone human interface guidelines. User experience. Apple, Inc., 11-21-2008.

http://developer.apple.com/iphone

Bersvendsen, Arve. Widgets 1.0 working draft. November 09, 2006. http://virtuelvis.com/archives/2006/11/widgets-10-working-draft

Burigat, Stefano; Chittaro, Luca; Gabrielli, Silvia. "Navigation techniques for small-screen devices: an evaluation on maps and web pages". Intl. journal of human-computer studies, February 2008, v. 66, n. 2, pp. 78-97.

Candelon, François; Arie, Sam. The mobile Web: a second chance. The Boston Consulting Group, 2007.

http://www.bcg.com/impact_expertise/publications/files/Mobile_Web_July_ 2007.pdf

Chakraborty, Dipanjan; Goyal, Sunil; Mittal, Sumit; Mukherjea, Sougata. "On the changing face of service composition in telecom". En: Proceedings of the 2007 Workshop on middleware for next-generation converged networks and applications (Port Beach, California, November 2630, 2007). Mncna'07. ACM, New York, NY, pp. 1-6.

Chan, Bryon; Flaherty, Jason. An architecture for fast AJAX enabled Web forms. Final report. Department of Electrical \& Computer Engineering, Part 4 Project report, 2005. University of Auckland, New Zealand.

Cobo-Romaní, Cristóbal; Pardo-Kuklinski, Hugo. Planeta Web 2.0. Inteligencia colectiva o medios fast food. Grup de Recerca d'Interaccions Digitals, Universitat de Vic, septiembre de 2007

http://www.planetaweb2.net

Crespo, Salvador. "Widgets por todas partes". Telefónica. Sociedad de la información, 9 de mayo de 2007.

http://sociedaddelainformacion.telefonica.es/documentos/articulos/B_ TECNOL_Widgets.pdf

De la Torre, Aníbal. Definición de Web 2.0. Blog de Aníbal de la Torre, 12 de abril de 2006.

http://www.adelat.org/index.php?title=conceptos_clave_en_la_web_2_0_ y_iii

Kleppmann, Martin. Increasing user satisfaction on the mobile web: technical considerations. Ept computing, diciembre de 2007.

http://www.eptcomputing.com/publications/mobile4web4satisfaction.pdf

Hoegg, Roman; Martignoni, Robert; Meckel, Miriam; Stanoevska, Katarina. Overview of business models for Web 2.0. Universität St. Gallen, Institute of Media and Communication Management, 2006.

http://c4mobile.ptinovacao.pt/files/GeNeMe2006.pdf

Maximilien, E. Michael. "Mobile mashups: thoughts, directions, and challenges". IEEE Intl. conf. on semantic computing, 2008, pp. 597-600. 
Mahemoff, Michael. AJAX design patterns: creating Web 2.0 sites with programming and usability patterns. O'Reilly, 2006.

Mäkeläinen, Sami; Alakoski, Timo. "Fixed-mobile hybrid mashups: applying the REST principles to mobile-specific resources". En: Proceedings of the 2008 Intl. workshops on web information systems engineering (Auckland, New Zealand, September 01-04, 2008). S. Hartmann; X. Zhou; M. Kirchberg, Eds. Lecture notes in computer science, 5176, pp. 172-182. Springer-Verlag, Berlin, Heidelberg. ISBN 978-3-540-85199-8

Mesbah, Ali; van Deursen, Arie. "An architectural style for AJAX". En: Wicsa '07: Proceedings of the 6th working IEEE/IFIP conf. on software architecture, pp. 44-53. IEEE Computer Society, 2007.

Munslow-Vilar, Bernard. Estudio, diseño e implementación de widgets. Universitat de Lleida, septiembre de 2007, p. 222.

http://www.recercat.net/bitstream/2072/4557/1/Munslow.pdf

Nielsen, Jakob. "Scrolling and scrollbars". Jakob Nielsen's Alertbox, Jul. 2005.

http://www.useit.com/alertbox/20050711.html

Nokia press releases. Nokia and 560 make the Web personal on your mobile. April 16, 2007.

http://www.nokia.com/A4136001?newsid $=1118790$

Nokia press releases. WidSets reaches 1 million registered users. May 25, 2007

http://www.nokia.com/A4136001?newsid $=1128924$

Open Mobile Alliance. xhtml Mobile profile 1.2 DTD. 31 Mar. 2008. http://www.openmobilealliance.org/Tech/DTD/xhtml-mobile12.dtd

O'Reilly, Tim. What is Web 2.0. Design patterns and bussiness models for the next generation of software. O'Reilly Media, Inc, 30 Sept. 2005. http://www.oreillynet.com/pub/a/oreilly/tim/news/2005/09/30/what-is-web20.html

Perry, Bill. "Adobe Flash player coming to iPhone - The facts". Flash devices, March 19, 2008.

http://www.flashdevices.net/2008/03/adobe-flash-player-coming-to-iphone. html

Rodríguez, Pablo; Mukherjee, Sarit; Rangarajan, Sampath. "Session level techniques for improving web browsing performance on wire- less links". En: Proceedings of the 13th intl. conf. on World Wide Web, Intl. World Wide Web Cons. archive, 2004, pp. 121-130.

Torniai, C.; Battle, S.; Cayzer, S. Sharing, discovering and browsing geotagged pictures on the Web. Multimedia Integration \& Communication Centre, Università di Firenze: Hewlett-Packard Development Company, 2007

Toyama, K.; Logan, R.; Roseway, A. "Geographic location tags on digital images". En: Proceedings of the Eleventh ACM intl. conf. on multimedia (Berkeley, CA, USA, Nov. 02-08, 2003). Multimedia'03. ACM, New York, pp. 156-166.

Trevor, Jonathan. "Doing the mobile mash". Computer, v. 41, n. 2, pp. 104-106, Feb. 2008

W3C. "Device independent authoring language (DIAL). W3C Working draft 27 July 2007". Kevin Smith (Ed.).

http://www.w3.org/TR/dial/

W3C. "Guía Breve de independencia de dispositivo".

http://www.w3c.es/Divulgacion/GuiasBreves/IndependenciaDispositivo

W3C. "Widgets 1.0: Packaging and configuration. W3C Working draft 22 December 2008". Marcos Cáceres (Ed.).

http://www.w3.org/TR/widgets/

Werbach, Kevin. "Syndication, the emerging model for business in the internet era". Harvard business review, v. 78 n. 3, pp. 84-93, 214. May-Jun 2000.

Ángel Hernández-García, Santiago Iglesias-Pradas, Julián Chaparro-Peláez, Félix-José Pascual-Miguel Escuela Técnica Superior de Ingenieros de Telecomunicación, Universidad Politécnica de Madrid.

Av. Complutense, s/n, 28040 Madrid.

angel.hernandez@upm.es

s.iglesias@upm.es

julian.chaparro@upm.es

felixjose.pascual@upm.es

http://www.tige.ior.etsit.upm.es

\section{¿Sabías que ZP/ tiene blog?}

\section{Se llama "Zona de notas"}

\section{http: //wWw.elprofesionaldelainformacion.com/notas/}

\section{En él puedes seguir toda la} actualidad y noticias sobre "El profesional de la información": novedades, comentarios sobre los contenidos, call for papers, reseñas de actividades, noticias sobre EPI en otros medios, etc.

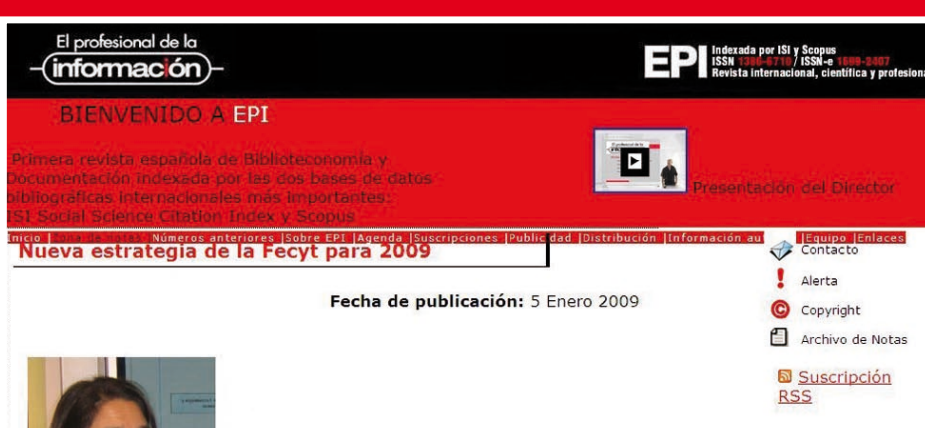

Cecilia Cabello, directora del Departamento de Gestión Integrada de la Información Científica, de la Fundación Española para la Ciencia y la Tecnología (Fecyt), presentó el nuevo plan estratégico de la misma en el Spanish Meeting Point de la Online Information Conference, Londres, 2-4 de diciembre de 2008. 